\title{
Close binary evolution and blue straggler formation
}

\author{
P. Lu. ${ }^{1,2} \dagger$, L. Deng ${ }^{1}$ \\ ${ }^{1}$ National Astronomical Observatories, Chinese Academy of Sciences, Beijing 100012, \\ P.R. China \\ email: lupin@bao.ac.cn \\ ${ }^{2}$ Graduate University of Chinese Academy of Sciences, Beijing, 100049, P.R. China
}

\begin{abstract}
In order to discuss the contribution of mass transfer in primordial close binaries to the blue straggler population in young clusters, we use Eggleton's stellar evolution code to simulate a grid of case A binary evolutionary models with the initial donor mass $2.0-8.0 \mathrm{M}_{\odot}$ and mass ratio $0.1-0.9$. The models cover the whole case A binaries that will experience mass transfer between 30.0 Myr to 1.0 Gyr. Based on such detailed models, we present a simulation to compare with the HST observation of young cluster NGC 1831 which can be fit with an isochrone of $\log ($ age $)=8.65$. The results show very few blue stragglers could be produced by case A binary evolution. There must be some other mechanisms for blue straggler formation in young clusters.
\end{abstract}

Keywords. Stars: blue stragglers, stars: binaries: close, Galaxy: open clusters and associations: individual (NGC 1831).

\section{Introduction}

Blue stragglers (BSs) are a kind of very special stars that lie on the extension of main sequence, in the area which is bluer and more luminous than the main sequence turn off (MSTO) in color magnitude diagram (CMD). Since the first observation of BSs in globular cluster M3 (Sandage 1953), they have been widely observed in all types of stellar systems by now. As the brightest and bluest "main sequence stars", they make a considerable contribution to the blue side of a stellar system's integrated spectrum energy distribution (ISED) (Deng et al. 1999, Schiavon et al. 2004, Xin \& Deng 2005).

Based on single stellar evolution theory, stars massive than the MSTO should already have evolved off the main sequence. BSs seem to be the stars which have longer life time for their mass or they formed later than the others in the cluster. Both the two reasons can not explain the existence of BSs very well. Several mechanisms have been proposed to explain BS formation and now it is widely accepted that BSs are formed in binaries by directly collision or primordial binary evolution.

Direct collision is usually related to dense environment in the cores of star clusters (Fregeau et al. 2004). It is supposed to be an important scnario of BSs formation in the dense cluster center. We name the BSs produced under this way dynamical blue stragglers (DBSs). Primordial binaries evolution is another mechanisms of BSs formation. Primordial binaries means the binaries that already exist when the cluster formed. It is highly affected by initial binary fraction. Most dynamical interactions in dense cluster cores tend to destroy binaries, so this mechanism is thought to be important in a sparse

$\dagger$ Present address: 20A Datun Road, Chaoyang District, Beijing, China(100012) 
environment (Mathys 1991). We call them primordial BSs (PBSs). The two mechanisms work at the same time in the evolution of stellar systems.

In 1964, McCrea proposed that BSs could be formed as the remnants of mass exchange in close binary systems. The massive star in the binary evolves faster, and mass transfer begins after the primary fills up its Roche lobe envelope. Then, as the hydrogen-rich material of the primary's envelope expanded out of the Roche lobe and transfers to the surface of the secondary through inner Lagrangian point, the companion will gradually grow in mass and become bluer and more luminous. As a result of mass exchange, the secondary becomes the massive one in the binary and will have a longer life time remaining on main sequence.

Mass transfer scnario can be divided into 3 subtypes - case A, case B and case C based on the evolution phase of the donor when mass transfer begins (Kippenhahn \& Weigert 1968). They are related to the time mass transfer begins when the donor is still on main sequence with a hydrogen burning core, it has evolved off the main sequence to the red giant branch before helium core ignition, or later evolutionary phase.

In this paper, we only focus on the mass transfer scnario and we limit our models in case A. A very detailed modelling of case A binary evolution is carried out in this paper. In order to investigate the distribution of BSs formed via case A mass transfer, we present a Mento-Carlo simulation to compare with the Hubble Space Telescope (HST) observations of young clusters. Young clusters are chosen so as to minimum the effect of dynamical evolution of stellar systems to BS formation. The calculation of our models is described in Sect. 2. and the result of Mento-Carlo simulation is given in Sect. 3 . Summary and conclusion is presented in the final section.

\section{The model of primordial blue stragglers}

We use Eggleton's stellar evolution code which has been updated (Han et al. 1994,2000 \& Pols et al. 1995,1998) to compute close binary evolution. The opacity library radiative (Iglesias \& Rogers 1996) and molecular opacities (Alexander \& Ferguson 1994) is adopted in this code.

So as to simplify the calculation, we take some assumptions which are reasonable in these models. The binary systems are assumed to be conservative in mass and angular momentum for no obvious mass loss in intermediate mass stars. The initial eccentricity $\mathrm{e}=0$ is adopted in the calculation. Tidal evolution is neglected because it has little influence on circular orbital binaries. Magnetic braking and stellar spins is also ignored for simplicity.

In the calculation, Roche lobe overflow (RLOF) is considered as a boundary condition with the mass transfer rate

$$
d m / d t=\text { const. } \times \max \left[0,\left(R_{\text {star }} / R_{\text {lobe }}-1\right)^{3}\right]
$$

where $R_{\text {star }}$ is the radius of the donor and $R_{\text {lobe }}$ is the Roche lobe radius (Eggleton 1983). We adopt the const. $=500 M_{\odot} y r^{-1}$ to keep a steady RLOF. Mass transfer history of the donor extracted from the calculation is recorded and then used as the input in a subsequent calculation of the companion. The material is assumed to be deposited on the surface of the secondary with zero falling velocity and homogeneously distributed over the outer layer. For the evolution of close binary, the formation of binary merger and subsequence evolution of a merger is not very certain so we manually terminate the code when binary systems contact or merge.

Figure 1 shows an example of case A binary evolution model with initial donor mass $2.9 M_{\odot}$, companion $2.6 M_{\odot}$, orbital separation $13.0 R_{\odot}$ and solar composition $[\mathrm{Z}=0.02$, 


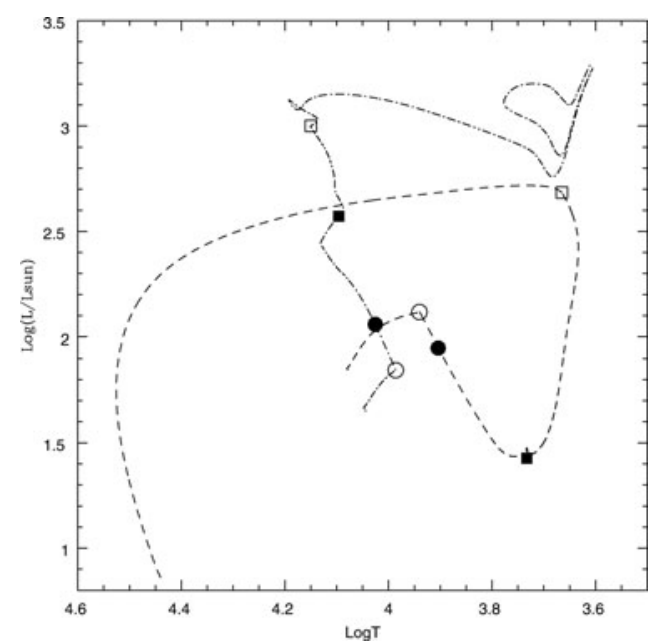

Figure 1. The evolutionary tracks of the two stars in our example binary. The dashed and dash-dotted lines are the evolutionary tracks of the donor and the accretor. The open circles, filled circles, filled squares and open squares on the tracks mean: mass transfer begins, mass ratio equals 1 , the system is 446.7 Myr old, and mass transfer terminates.

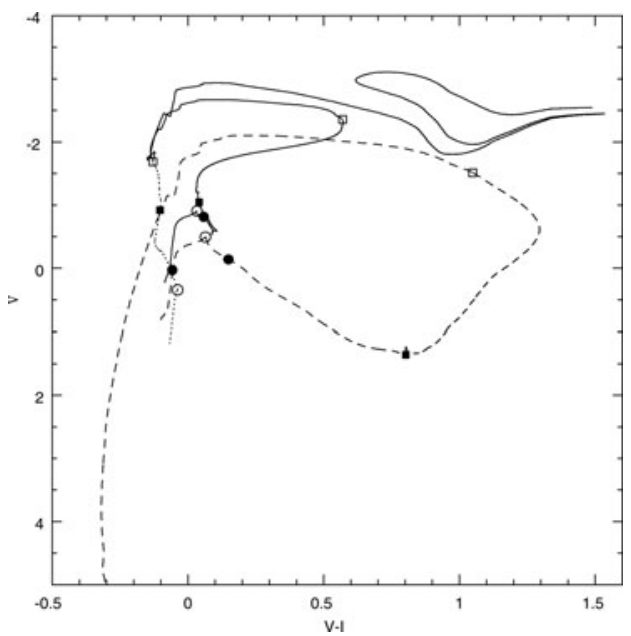

Figure 2. The synthetic evolutionary track of the two components in our example binary system. The solid line is the synthetic evolutionary track of the two components. The dashed and dotted lines are the evolutionary tracks of the donor and the accretor respectively. All the marks means the same as in Fig. 1.

Table 1. Main results of the example binary $\left(2.9 M_{\odot}+2.6 M_{\odot}\right)$

\begin{tabular}{|c|c|c|c|c|c|c|c|c|c|}
\hline Epoch & Age $\left(10^{9} \mathrm{yrs}\right)$ & $P(d)$ & $a\left(R_{\odot}\right)$ & $\operatorname{Mass}\left(M_{\odot}\right)$ & $\lg \left(L / L_{\odot}\right)$ & $\lg T_{e f f}$ & $X_{C}$ & $Y_{C}$ & $\dot{M}\left(M_{\odot} y r^{-1}\right)$ \\
\hline \multirow[t]{2}{*}{1} & 0.0000 & 2.3161 & 13.0000 & 2.9000 & 4.0781 & 1.8437 & 0.700 & 0.280 & 0.0 \\
\hline & & & & 2.6000 & 4.0433 & 1.6495 & 0.700 & 0.280 & \\
\hline \multirow[t]{2}{*}{2} & 0.3896 & 2.3161 & 13.0000 & 2.9000 & 3.9402 & 2.1169 & 0.089 & 0.892 & 0.0 \\
\hline & & & & 2.6000 & 3.9861 & 1.8452 & 0.336 & 0.645 & \\
\hline \multirow[t]{2}{*}{3} & 0.3906 & 2.2957 & 12.9237 & 2.7500 & 3.9040 & 1.9499 & 0.086 & 0.895 & $9.1768 \mathrm{E}-07$ \\
\hline & & & & 2.7500 & 4.0256 & 2.0584 & 0.337 & 0.644 & \\
\hline \multirow[t]{2}{*}{4} & 0.4467 & 4.2559 & 19.5029 & 1.5640 & 3.7329 & 1.4268 & 0.015 & 0.966 & $2.7073 \mathrm{E}-09$ \\
\hline & & & & 3.9207 & 4.0960 & 2.5719 & 0.249 & 0.732 & \\
\hline \multirow[t]{2}{*}{5} & 0.4761 & 117.476 & 178.1303 & 0.3993 & 3.6652 & 2.6840 & 0.000 & 0.981 & 0.0 \\
\hline & & & & 5.0852 & 4.1500 & 3.0004 & 0.112 & 0.869 & \\
\hline
\end{tabular}

The columns are (1) model serial number, (2) age, (3) period, (4) orbital separation, (5) mass,

(6) luminosity, (7) effective temperature, (8) hydrogen abundance in the core, (9) helium abundance in the core, (10) mass transfer rate.

$\mathrm{Y}=0.28]$. Roche lobe mass transfer of the donor begins at $\log ($ age $)=8.59$ and then the secondary becomes more and more massive. At the meanwhile, it climbs up on the extension of main sequence and gradually become bluer and more luminous on HR diagram. Our comparison sample young cluster NGC 1831 can be fit with an isochrone of $\log ($ age $)=8.65$, so we also mark the position of the two components at that age with filled square on Fig 1. When mass transfer terminate, the donor becomes a red giant with only $0.4 M_{\odot}$. The helium core can not be ignited and it will quickly turn to a helium white dwarf. As a result of mass transfer, the secondary remains on main sequence with a hydrogen burning core. The parameters of the binaries at some epoches are listed in Table 1 . 
Usually, close binaries especially those belongs to case A cannot be visually resolved in observations, so we somehow need to get the synthetic spectrum energy distribution of the two components in a binary system. For the HST observation of NGC 1831, it is convenient to convert the magnitude in F555 and F814 band to standard V and I magnitude. So we also need to get the synthetic Johnson-Cousins V and I magnitude of all our models by convolving correspond filter response.

A standard spectrum library for evolutionary synthesis has been presented by Lejeune et al. $(1997,1998)$ and they also obtained the colors and bolometric corrections, synthesized in the $(U B V)_{J}(R I)_{C} J K L L^{\prime} M$ system. The library covers wide ranges of fundamental parameters: $T_{\text {eff }}: 50000 \mathrm{~K} \sim 2000 \mathrm{~K}, \log g: 5.5 \sim-1.02$ and $[\mathrm{M} / \mathrm{H}]:+1.0 \sim-5.0$. Given effective temperature, surface gravity and solar composition, we can easily get the Johnson-Cousins $\mathrm{V}$ and I magnitude of all our models by an interpolation. The evolutionary tracks of the example binary in CMD are shown in Fig. 2. The synthetic evolutionary track is also shown in this figure. The evolution history of synthetic color V-I is given in Fig. 3. Mass ratio becomes 1 right after mass transfer begins. After that, the synthetic color is dominated by the secondary and move slowly to the blue side. As a result of mass exchange, the synthetic color can remain in the blue region for a longer time. As the donor climbs up along the Hayashi line and expands it's envelope, the color will quickly evolve to the red side. Mass transfer keeps on going until the donor begins contract at the top of Hayashi line and then the secondary dominates the color again. The synthetic model will evolve to the very blue and luminous region and remain there for about $10 \mathrm{Myr}$ and then follow the evolution of the companion.

Blue stragglers formed via mass transfer in old open clusters M67 has been studied (Tian et al. 2006). So we focus on case A binaries in young clusters. To simulate a continuous process in the evolution of young clusters, we computed a grid of close binary evolution models with an initial intermediate donor mass from 2.0 to $8.0 M_{\odot}$. Mass ratio is from 0.1 to 0.9 with 0.1 interval and the orbital separation covers the whole case A. The models cover the whole case A binary evolution that will experience mass transfer between 30.0 Myr to 1.0 Gyr. A 'snapshot' of all these models in the CMD at $\log ($ age) $=8.65$ are taken for the subsequent Mento-Carlo simulation.

\section{Mento-Carlo simulations of the primordial BSs in NGC 1831}

In order to investigate the distribution of BSs formed via close binary mass transfer in young clusters and compare with observations. We choose NGC 1831 as a sample to perform our Mento-Carlo simulation. The cluster can be fit with an isochrone of $\log ($ age $)=$ 8.65 with solar composition.

\subsection{Initial parameters of the cluster NGC 1831}

To simulate a cluster for the comparison with observations, we adopt the initial parameters of the cluster shown below.

1) Initial mass function

We adopt the initial mass function given by Kroupa, Tout \& Gilmore $(1990,1991)$. They found a mass generating function following Eggleton (Eggleton, Fitchett \& Tout 1989),

$$
M(X)=0.33\left[\frac{1}{(1-X)^{0.75}+0.04(1-X)^{0.25}}-\frac{1}{1.04}(1-X)^{2}\right]
$$

where $\mathrm{X}$ is a random number from 0 to 1 and $\mathrm{M}$ is the total mass of the two components in a binary system. 


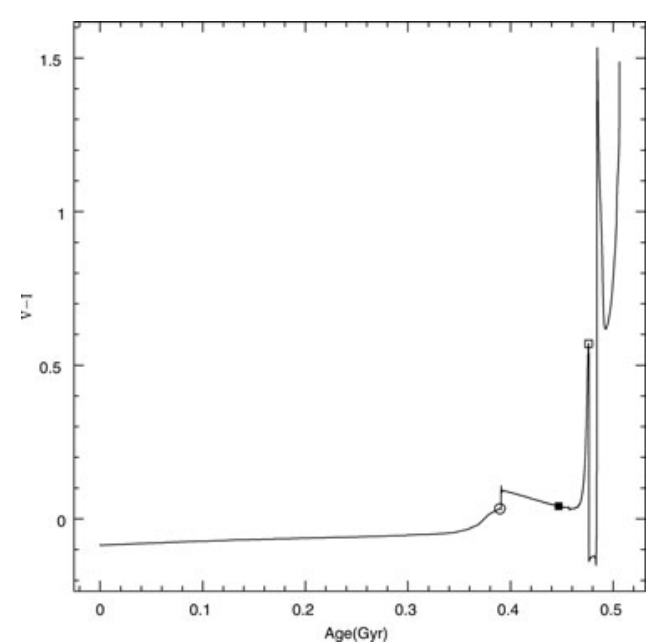

Figure 3. Evolution history of the synthetic color of the example binary system. The open circle, filled square and open square means: mass transfers begins, the system is $446.7 \mathrm{Myr}$ old and mass transfer terminates.

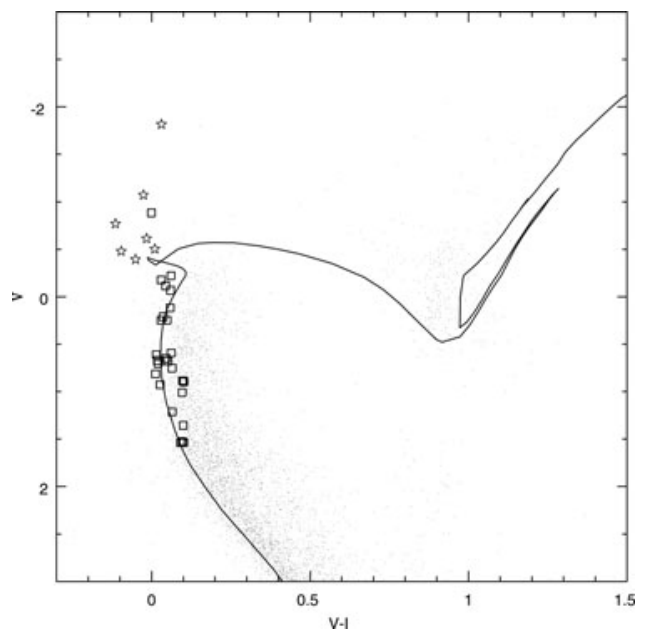

Figure 4. The solid line is the 446.7 Myr theoretical isochrone. The open asterisks are the high possibility $\mathrm{BS}$ candidates and the open squares are the 28 binaries from our simulation.

\section{2) Initial mass ratio}

We adopt a uniform distribution of initial mass ratio (Hurley, Tout, Aarseth \& Pols 2001).

$$
1>q>\max [0.1(M(X)-0.1), 0.02(M(X)-50.0)]
$$

where the $\mathrm{M}$ is the total mass of a binary from the initial mass function and limited to 0.1-50.0 $M_{\odot}$.

3) Initial orbital separation

A flat distribution of initial orbital separation from Pols \& Marinus (1994) are used here.

$$
\Gamma(\alpha) \propto \alpha^{-1}, \alpha_{\max }>\alpha>\alpha_{\min }, \alpha_{\max } / \alpha_{\min } \approx 5000
$$

where $\alpha=R / D$. $\mathrm{R}$ is the radius of stars and $\mathrm{D}$ is the orbital separation. $\alpha_{\text {min }}$ is the minimum size of the Roche lobe that just fits a zero-age star and $\alpha_{\max } / \alpha_{\min } \approx 5000$ corresponds to $100 \mathrm{AU}$ orbital separation for a $2 M_{\odot}$ primary which makes sure that about two thirds of all binaries are close and will interact at some evolutionary stage.

In the CMD of NGC 1831, we pay more attention to the region from MSTO to 2 mag below, which limits the initial donor mass $2.0-3.2 M_{\odot}$. The initial binary fraction in this region is set to be $50 \%$. Finally, we obtain 772 primordial binaries with initial donor mass $2.0-3.2 M_{\odot}$ using the distribution of initial parameters listed above.

\subsection{Results}

After an interpolation in the precious 'snapshot' we have gotten, only 28 binaries can be interpolated and their positions in the CMD are shown in Fig 4 with open square. The points marked with asterisk are the high possibility candidate of BSs in the observation. Among the 28 binaries, only 1 is bluer and more luminous than MSTO thus could be a primordial blue straggler. 


\section{Summary and conclusion}

The simulation shows very few BSs could be predicted by case A binary evolution in young clusters. There is a severe lack of BSs if we only consider mass transfer scnario in case A binaries. Based on the data of our models, the reason could be:

1. The distribution of initial parameters in simulation, especially the orbital separation. A flat distribution of initial orbital separation with an upper limit $\alpha_{\text {max }} \approx 5000 \alpha_{\text {min }}$ can only give about $10 \%$ case A binaries. More of them belong to case $\mathrm{B}$ and $\mathrm{C}$.

2. Coalescence. The evolutions of contact binaries and mergers are very important for case A. During the evolution, case A binaries could easily contact or merge especially for those with higher donor mass, small or intermediate mass ratio and small orbital separation. So, at $\log ($ age $)=8.65$, a significant portion of our close binary models have already contacted or merged.

Then as a result, finally, only $3 \%-4 \%$ of the total binaries can be interpolated thus even fewer blue stragglers can be produced via case A binary evolution. So, case A binary mass transfer might not be the main mechanism of BSs formation in young clusters. Coalescence and case B binary evolution needs to be considered or other mechanisms need to be introduced to the formation of blue stragglers in young clusters such as collision and so on.

\section{Acknowledgements}

We would like to thank Q. Liu for the data reduction of NGC 1831 and providing us the data. We also would like to acknowledge B. Tian for writing a document on how to use Eggleton's stellar evolution code.

\section{References}

Alexander, D. R. \& Ferguson, J. W. 1994, ApJ 437, 879

Chen, X. F. \& Han, Z. W. 2004, MNRAS 355, 1182

Deng, L., Chen, R., Liu, X. S., \& Chen, J. S. 1999, ApJ 524, 824

Eggleton, P. P. 1971, MNRAS 151, 351

Eggleton, P. P. 1972, MNRAS 156, 361

Eggleton, P. P. 1973, MNRAS 163, 279

Han, Z., Podsiadlowski, Ph., \& Eggleton, P. P. 1994, MNRAS 270, 121

Han, Z., Tout, C. A., \& Eggleton, P. P. 2000, MNRAS 319, 215

Hurly, J. R., Tout, C. A., Aarseth, S. J., \& Pols, O. R. 2001, MNRAS 323, 630

Hurly, J. R., Pols, O. R., Aarseth, S. J., \& Tout, C. A. 2001, MNRAS 363, 293

Iglesias, C. A. \& Rogers, F. J. 1996, ApJ 464, 943

Kroupa, P., Tout, C. A., \& Gilmore, G. 1991, MNRAS 251, 293

Lejeune, Th., Cuisinier, F., \& Buser, R. 1997, A\&AS 125, 229

Lejeune, Th., Cuisinier, F., \& Buser, R. 1998, A\&AS 130, 65

McCrea, W. H. 1964, A\&SA 128, 147

Pols, O. R. \& Marinus, M. 1994, A\&A 288, 475

Pols, O. R., Tout, C. A., Eggleton, P. P., \& Han, Z. 1995, MNRAS 274, 964

Pols, O. R., Schroder, K.-P., Hurley, J. R., Tout, C. A., \& Eggleton, P. P. 1998, MNRAS 298, 525

Sandage, A. R. 1953, AJ 58, 61

Tian, B., Deng, L., Han, Z., \& Zhang, X. B. 2006, A\&AS 455, 247

Xin, Y. \& Deng, L. 2005, ApJ 619, 824 


\section{Discussion}

K. StePEIN: If I understand correctly, your model predicts that BSs should be Algols?

P. Lu: Yes, most BSs formed via case A should be Algols.

K. STEPEIn: A lack of a sufficient number of close binaries which can transform into BSs may result from neglecting angular momentum loss.

P. LU: More binaries could become close during the evolution by considering angular momentum loss, but at the meanwhile, the disadvantage is that more close binaries will merge by taking account of momentum loss. We can not tell exactly the subsequent evolution.

O. Plos: An explanation for the small number of blue stragglers formed by case A mass transfer in your models, could be that most blue stragglers in young clusters are actually formed by case B mass transfer. In rather massive binaries the parameter space for case $\mathrm{B}$ is larger than for case A. This would also explain the lack of Algols among observed blue stragglers, because case B mass transfer produces wide, detached remnants.

J. Christensen-DalsgandD: Blue stragglers in old clusters can be in the Cepheid instability strip; in young clusters they may be $\beta$ cephei stars. In these cases observations of oscillations could constrain the internal structure. Do the different formation scenarios yield different internal structures that we could tell apart with astero-seismology.

P. Lu: In fact, BSs in our models are binaries. Their synthetic positions on CMD may in the Cepheid instability strip, but actually they may not be $\beta$ cephei stars respectively. BSs formed via directly collision or merger may be different, but we still could not exactly describe the detailed internal structure. 